\title{
Fast homoclinic solutions for damped vibration problems with superquadratic potentials
}

Xinhe Zhu ${ }^{1 *}$ and Ziheng Zhang ${ }^{1}$

\section{"Correspondence:}

zhumath@126.com

${ }^{1}$ School of Mathematical Sciences,

Tianjin Polytechnic University,

Tianjin, P.R. China

\begin{abstract}
In this paper we investigate the existence and multiplicity of homoclinic solutions for the following damped vibration problem:

$$
\ddot{u}+q(t) \dot{u}-L(t) u+W_{u}(t, u)=0,
$$

where $q: \mathbb{R} \rightarrow \mathbb{R}$ is a continuous function, $L \in C\left(\mathbb{R}, \mathbb{R}^{n^{2}}\right)$ is a symmetric and positive definite matrix for all $t \in \mathbb{R}$ and $W \in C^{1}\left(\mathbb{R} \times \mathbb{R}^{n}, \mathbb{R}\right)$. The novelty of this paper is that, assuming $\lim _{|t| \rightarrow+\infty} Q(t)=+\infty\left(Q(t)=\int_{0}^{t} q(s) d s\right)$ and $L$ is coercive at infinity, we establish one new compact embedding theorem. Subsequently, supposing that $W$ satisfies the global Ambrosetti-Rabinowitz condition, we obtain some new criterion to guarantee the existence of homoclinic solution of (DS) using the mountain pass theorem. Moreover, if $W$ is even, then (DS) has infinitely many homoclinic solutions. Recent results in the literature are generalized and significantly improved.
\end{abstract}

MSC: 34C37; 35A15; 35B38

Keywords: Homoclinic solutions; Critical point; Variational methods; Mountain pass theorem

\section{Introduction}

The purpose of this work is to deal with the existence of homoclinic solutions for the following damped vibration problem:

$$
\ddot{u}+q(t) \dot{u}-L(t) u+W_{u}(t, u)=0,
$$

where $q: \mathbb{R} \rightarrow \mathbb{R}$ is a continuous function such that

$$
\lim _{|t| \rightarrow+\infty} Q(t)=+\infty
$$

with $Q(t)=\int_{0}^{t} q(s) d s, L \in C\left(\mathbb{R}, \mathbb{R}^{n^{2}}\right)$ is a symmetric and positive definite matrix for all $t \in \mathbb{R}$ and $W \in C^{1}\left(\mathbb{R} \times \mathbb{R}^{n}, \mathbb{R}\right)$. 
When $q(t) \equiv 0$, (DS) is just the following second order Hamiltonian system:

$$
\ddot{u}-L(t) u+W_{u}(t, u)=0 .
$$

It is well known that the existence of homoclinic solutions for Hamiltonian systems and their importance in the study of the behavior of dynamical systems have been recognized from Poincaré [16]. They may be "organizing centers" for the dynamics in their neighborhood. From their existence one may, under certain conditions, infer the existence of chaos nearby or the bifurcation behavior of periodic orbits. In the past two decades, with the works of [15] and [18], variational methods and critical point theory have been successfully applied for the search of the existence and multiplicity of homoclinic solutions of (HS). Assuming that $L(t)$ and $W(t, u)$ are independent of $t$ or $T$-periodic in $t$, many authors have studied the existence of homoclinic solutions for the Hamiltonian system (HS) (see, for instance, $[3,6,8,18,26]$ and the references therein) and some more general Hamiltonian systems are considered in the recent papers [10, 12, 22]. In this case, the existence of homoclinic solutions can be obtained by going to the limit of periodic solutions of approximating problems.

If $L(t)$ and $W(t, u)$ are neither autonomous nor periodic in $t$, the existence of homoclinic solutions of (HS) is quite different from the periodic systems because of the lack of compactness of the Sobolev embedding, see for instance $[1,11,15,19]$ and the references therein. It is worthy of pointing out that to obtain the existence of homoclinic solutions of (HS), the following so-called global Ambrosetti-Rabinowitz condition ((AR) condition, see $\left(\mathrm{W}_{1}\right)$ below) on $W$ due to Ambrosetti-Rabinowitz (e.g., [2]) is assumed in the works mentioned above, which implies that $W(t, u)$ is of superquadratic growth as $|u| \rightarrow+\infty$. However, there are lots of potentials which are superquadratic as $|u| \rightarrow+\infty$ but do not satisfy the (AR) condition. Therefore, many authors have been focusing their attention on obtaining the existence of homoclinic solutions under the conditions weaker than the (AR) condition, see for instance $[7,13,14,23,32]$ and the references listed therein. In addition, to verify the (PS) condition for the corresponding energy functional of (HS), the following coercive assumption on $L$ is often needed:

(L) $L \in C\left(\mathbb{R}, \mathbb{R}^{n^{2}}\right)$ is a symmetric and positive definite matrix for all $t \in \mathbb{R}$, and there is a continuous function $\beta: \mathbb{R} \rightarrow \mathbb{R}$ such that $\beta(t)>0$ for all $t \in \mathbb{R}$ and $(L(t) u, u) \geq$ $\beta(t)|u|^{2}$ and $\beta(t) \rightarrow+\infty$ as $|t| \rightarrow+\infty$,

which indicates that the smallest eigenvalue $l(t)$ of $L(t)$ is coercive, i.e.,

$$
l(t) \rightarrow+\infty \quad \text { as }|t| \rightarrow+\infty,
$$

where $l(t):=\inf _{|u|=1}(L(t) u, u),(\cdot, \cdot): \mathbb{R}^{n} \times \mathbb{R}^{n} \rightarrow \mathbb{R}$ denotes the standard inner product in $\mathbb{R}^{n}$ and subsequently $|\cdot|$ is the induced norm.

Compared with the literature available for $W(t, u)$ being superquadratic as $|u| \rightarrow+\infty$, the study of the existence of homoclinic solutions of (HS) under the assumption that $W(t, u)$ is subquadratic at infinity is much more recent and the number of references is considerably smaller, see for instance $[8,20,21,28,29]$, where some other types of coercive conditions on $L$ are also utilized. In addition, the existence of homoclinic solutions under the condition that $W(t, u)$ is asymptotically quadratic at infinity has also been investigated by many researchers, see for instance $[9,24,30,32]$. 
As far as the case that $q(t) \neq 0$ is concerned, to our best knowledge, there is little research about the existence of homoclinic solutions of (DS). In the recent paper [31], for the first time the authors investigated the existence of fast homoclinic solutions for the following special case of (DS):

$$
\ddot{u}+c \dot{u}-L(t) u+W_{u}(t, u)=0, \quad t \in \mathbb{R}, u \in \mathbb{R}^{n},
$$

with $W\left(W(t, u)=a(t)|u|^{\gamma}, 1<\gamma<2\right)$ is subquadratic at infinity and $c \geq 0$ via a standard minimizing argument, which has been improved in [4] where, using the genus property in critical point theory, the authors considered the case that $q$ satisfies $(1.1)$ and $W(t, u)$ is of subquadratic growth and obtained the existence of infinitely many fast homoclinic solutions of (DS). In addition, in [25], the authors studied the existence of solutions for the following damped vibration problems:

$$
\left\{\begin{array}{l}
\ddot{u}(t)+q(t) u(t)=L(t) u(t)+W_{u}(t, u), \quad \text { a.e. } t \in[0, T], \\
u(0)-u(T)=\dot{u}(0)-e^{Q(T)} \dot{u}(T)=0,
\end{array}\right.
$$

using the variational methods, where $Q(T)=\int_{0}^{T} q(s) d s$.

Motivated by the above papers, in this paper we use the mountain pass theorem to establish some new criterion to guarantee (DS) has infinitely many fast homoclinic solutions for the case that $W$ satisfies the (AR) condition. In the following, in order to introduce the concept of fast homoclinic solutions of (DS) conveniently, we firstly describe some properties of the weighted Sobolev space $E$ on which the energy functional associated with (DS) is defined. Letting

$$
E=\left\{u \in H^{1}\left(\mathbb{R}, \mathbb{R}^{n}\right): \int_{\mathbb{R}} e^{Q(t)}\left[|\dot{u}(t)|^{2}+(L(t) u(t), u(t))\right] d t<+\infty\right\},
$$

where $Q(t)$ is defined in (1.1), and for any $u, v \in E$, define

$$
(u, v)_{E}=\int_{\mathbb{R}} e^{Q(t)}[(\dot{u}(t), \dot{v}(t))+(L(t) u(t), v(t))] d t .
$$

Then the space $E$ is a Hilbert space with the above inner product, and the corresponding norm is

$$
\|u\|=\left\{\int_{\mathbb{R}} e^{Q(t)}\left[|\dot{u}(t)|^{2}+(L(t) u(t), u(t))\right] d t\right\}^{1 / 2} .
$$

Here, $H^{1}\left(\mathbb{R}, \mathbb{R}^{n}\right)$ denotes the Banach spaces of functions on $\mathbb{R}$ with values in $\mathbb{R}^{n}$ under the norm

$$
\|u\|_{H^{1}}:=\left(\|u\|_{2}^{2}+\|\dot{u}\|_{2}^{2}\right)^{1 / 2}
$$

Throughout this paper, we adopt the definition of [4] for fast homoclinic solution of (DS): 
Definition 1.1 If (1.1) holds, a solution $u$ of (DS) is called a fast homoclinic solution if $u \in E$.

In what follows, we can state our main result. For the convenience of statement, $W(t, u)$ is assumed to satisfy the following conditions:

$\left(\mathrm{W}_{1}\right)$ There is a constant $\mu>2$ such that, for every $t \in \mathbb{R}$ and $u \in \mathbb{R}^{n} \backslash\{0\}$,

$$
0<\mu W(t, u) \leq\left(W_{u}(t, u), u\right)
$$

$\left(\mathrm{W}_{2}\right) \quad W_{u}(t, u)=o(|u|)$ as $|u| \rightarrow 0$ uniformly with respect to $t \in \mathbb{R}$;

$\left(\mathrm{W}_{3}\right)$ There exists $\bar{W} \in C\left(\mathbb{R}^{n}, \mathbb{R}\right)$ such that $\left|W_{u}(t, u)\right| \leq|\bar{W}(u)|$ for every $t \in \mathbb{R}$ and $u \in \mathbb{R}^{n}$.

Theorem 1.2 Suppose that (1.1), (L), and $\left(\mathrm{W}_{1}\right)-\left(\mathrm{W}_{3}\right)$ are satisfied, then (DS) has at least one nontrivial fast homoclinic solution. Moreover, if we assume that $W(t, u)$ is even in $u$, i.e.,

$\left(\mathrm{W}_{4}\right) W(t, u)=W(t,-u)$ for all $t \in \mathbb{R}$ and $u \in \mathbb{R}^{n}$,

then (DS) possesses infinitely many distinct fast homoclinic solutions.

Remark 1.3 In (DS), if $q(t) \equiv 0$, then Theorem 1.2 (under the same hypothesis on $L$ and $W(t, u)$ ) reaches the results in [15](see its Theorem 1 and Theorem 2). Therefore, we extend the results of [15] for (HS) to the more general situations (DS).

It is worth pointing out that an open problem was proposed in [31], explicitly, how to obtain the existence of fast homoclinic solutions of (1.3) for the case that $W$ satisfies the (AR) condition using the mountain pass theorem. Here, Theorem 1.2 gives some partial answer to this open problem. For some recently related results, we refer the reader to $[5,27]$.

Remark 1.4 From (L), it is easy to obtain that there exists a constant $\beta>0$ such that

$$
(L(t) u, u) \geq \beta|u|^{2}, \quad \forall(t, u) \in \mathbb{R} \times \mathbb{R}^{n} .
$$

$\left(\mathrm{W}_{1}\right)$ is called the global Ambrosetti-Rabinowitz condition due to Ambrosetti and Rabinowitz (see [2]), which implies that

$$
\begin{aligned}
& W(t, u) \leq W\left(t, \frac{u}{|u|}\right)|u|^{\mu}, \quad 0<|u| \leq 1, \\
& W(t, u) \geq W\left(t, \frac{u}{|u|}\right)|u|^{\mu}, \quad|u| \geq 1 .
\end{aligned}
$$

In fact, it suffices to show that for every $u \neq 0$ and $t \in \mathbb{R}$ the function $(0,+\infty) \ni \xi \rightarrow$ $W\left(t, \xi^{-1} u\right) \xi^{\mu}$ is non-increasing, which is an immediate consequence due to $\left(\mathrm{W}_{1}\right)$. Moreover, choose $\eta(t)=\min _{|u|=1} W(t, u)>0$, one has

$$
W(t, u) \geq \eta(t)|u|^{\mu}
$$


for every $t \in \mathbb{R}$ and $|u| \geq 1$. In addition, by $\left(\mathrm{W}_{1}\right)$ and $\left(\mathrm{W}_{2}\right)$, we have $W(t, u)=o\left(|u|^{2}\right)$ as $|u| \rightarrow 0$ uniformly with respect to $t \in \mathbb{R}$, i.e., for any $\epsilon>0$, there is $\delta>0$ such that

$$
W(t, u) \leq \epsilon|u|^{2} \quad \text { for }(t, u) \in \mathbb{R} \times \mathbb{R}^{n},|u| \leq \delta
$$

Furthermore, by $\left(\mathrm{W}_{2}\right)$ and $\left(\mathrm{W}_{3}\right)$, for any $u \in \mathbb{R}^{n}$ such that $|u| \leq r$, there exists some constant $d$ (dependent on $r$ ) such that

$$
\left|W_{u}(t, u)\right| \leq d|u|, \quad \forall t \in \mathbb{R},|u| \leq r .
$$

In what follows, we present some examples for $q(t), L(t)$, and $W(t, u)$ satisfying (1.1), (L), and $\left(W_{1}\right)-\left(W_{3}\right)$ to demonstrate our Theorem 1.2. Let us choose

$$
q(t)=t, L(t)=\left(1+t^{2}\right) I_{n}, \quad W(t, u)=\left(2+\sin ^{2} t\right)|u|^{\mu},
$$

where $I_{n}$ is the $n \times n$ identity matrix and $\mu>2$ is a constant. Then it is easy to check that all the hypotheses of Theorem 1.2 are satisfied.

The remaining part of this paper is organized as follows. Some preliminary results are presented in Sect. 2. In Sect. 3, we are devoted to accomplishing the proof of our main result.

\section{Preliminary results}

The main difficulty in dealing with the existence of infinitely many homoclinic solutions for (DS) is the lack of compactness of the Sobolev embedding. To overcome this difficulty under the assumptions of Theorem 1.2, we employ the following compact embedding theorem. For the statement convenience, define the function space $L^{2}\left(e^{Q(t)}\right)$ as the Banach space of functions on $\mathbb{R}$ with values in $\mathbb{R}^{n}$ under the norm

$$
\|u\|_{2, Q}:=\left(\int_{\mathbb{R}} e^{Q(t)}|u(t)|^{2} d t\right)^{1 / 2}
$$

It is obvious that $E \subset L^{2}\left(e^{Q(t)}\right)$ with the embedding continuous, i.e., there is a constant $C>0$ such that

$$
\|u\|_{2, Q} \leq C\|u\|, \quad \forall u \in E .
$$

In fact, we have the following compact embedding lemma.

Lemma 2.1 Suppose that (1.1) holds and L satisfies $(\mathrm{L})$, then the embedding of E in $L^{2}\left(e^{Q(t)}\right)$ is compact.

Proof For any $R>0$, define

$$
\zeta(R)=\inf _{|u|=1,|t| \geq R}(L(t) u, u) \geq \inf _{|t| \geq R} l(t) .
$$

By $(\mathrm{L}), \zeta(R) \rightarrow+\infty$ as $R \rightarrow+\infty$. 
Let $K \subset E$ be a bounded set, then there exists some $M>0$ such that $\|u\| \leq M$ for all $u \in K$. We show that $K$ is precompact in $L^{2}\left(e^{Q(t)}\right)$. For any $\epsilon>0$, take $R_{0}$ large enough such that

$$
\frac{4 M^{2} C^{2}}{\zeta(R)}<\frac{\epsilon^{2}}{2}, \quad \forall R \geq R_{0}
$$

By the Sobolev compact embedding theorem, $\left.E\right|_{(-R, R)}$ is compactly embedded in $\left.L^{2}\left(e^{Q(t)}\right)\right|_{(-R, R)}$ for all $R>0$. Hence, there are $u_{1}, \ldots, u_{m} \in K$ such that, for any $u \in K$, there is $u_{i}(1 \leq i \leq m)$ satisfying

$$
\int_{|t| \leq R_{0}} e^{Q(t)}\left|u-u_{i}\right|^{2} d t<\frac{\epsilon^{2}}{2}
$$

and so

$$
\begin{aligned}
\left\|u-u_{i}\right\|_{2, Q}^{2} & =\int_{|t| \leq R_{0}} e^{Q(t)}\left|u-u_{i}\right|^{2} d t+\int_{|t|>R_{0}} e^{Q(t)}\left|u-u_{i}\right|^{2} d t \\
& <\frac{\epsilon^{2}}{2}+\int_{|t|>R_{0}} e^{Q(t)} \frac{\left(L(t)\left(u-u_{i}\right), u-u_{i}\right)}{l(t)} d t \\
& \leq \frac{\epsilon^{2}}{2}+\frac{C^{2}}{\zeta\left(R_{0}\right)}\left\|u-u_{i}\right\|^{2} \\
& \leq \frac{\epsilon^{2}}{2}+\frac{4 M^{2} C^{2}}{\zeta\left(R_{0}\right)}<\epsilon^{2},
\end{aligned}
$$

which implies that $K$ has a finite $\epsilon$-net and, consequently, it is precompact in $L^{2}\left(e^{Q(t)}\right)$.

In order to obtain the existence of homoclinic solutions of (DS), we also need the following inequality. Denote by $L^{\infty}\left(\mathbb{R}, \mathbb{R}^{n}\right)$ the Banach space of essentially bounded functions from $\mathbb{R}$ into $\mathbb{R}^{n}$ equipped with the norm

$$
\|u\|_{\infty}:=\operatorname{ess} \sup \{|u(t)|: t \in \mathbb{R}\}
$$

Then we have

Lemma 2.2 ([4, Lemma 2.1]) For $u \in E$, one has

$$
\|u\|_{\infty} \leq \frac{1}{\sqrt{2 e_{0} \sqrt{\beta}}}\|u\|
$$

where $\beta$ is defined in (1.5) and $e_{0}=\min _{t \in \mathbb{R}} e^{Q(t)}$.

Now we introduce more notations and some necessary definitions. Let $\mathcal{B}$ be a real Banach space, $I \in C^{1}(\mathcal{B}, \mathbb{R})$, which means that $I$ is a continuously Fréchet-differentiable functional defined on $\mathcal{B}$. Recall that $I \in C^{1}(\mathcal{B}, \mathbb{R})$ is said to satisfy the (PS) condition if any sequence $\left\{u_{j}\right\}_{j \in \mathbb{N}} \subset \mathcal{B}$, for which $\left\{I\left(u_{j}\right)\right\}_{j \in \mathbb{N}}$ is bounded and $I^{\prime}\left(u_{j}\right) \rightarrow 0$ as $j \rightarrow+\infty$, possesses a convergent subsequence in $\mathcal{B}$. 
Moreover, let $B_{\rho}$ be the open ball in $\mathcal{B}$ with the radius $\rho$ and centered at 0 and $\partial B_{\rho}$ denote its boundary. To obtain the existence and multiplicity of fast homoclinic solutions of (DS), we appeal to the following well-known mountain pass theorem, see [17].

Lemma 2.3 ([17, Theorem 2.2$])$ Let $\mathcal{B}$ be a real Banach space and $I \in C^{1}(\mathcal{B}, \mathbb{R})$ satisfying the (PS) condition. Suppose that $I(0)=0$ and

(A1) there are constants $\rho, \alpha>0$ such that $\left.I\right|_{\partial B_{\rho}} \geq \alpha$, and

(A2) there is $e \in \mathcal{B} \backslash \bar{B}_{\rho}$ such that $I(e) \leq 0$.

Then I possesses a critical value $c \geq \alpha$. Moreover, $c$ can be characterized as

$$
c=\inf _{g \in \Gamma} \max _{s \in[0,1]} I(g(s)),
$$

where

$$
\Gamma=\{g \in C([0,1], \mathcal{B}): g(0)=0, g(1)=e\} .
$$

Lemma 2.4 ([17, Theorem 9.12]) Let $\mathcal{B}$ be an infinite dimensional real Banach space, and let $I \in C^{1}(\mathcal{B}, \mathbb{R})$ be even, satisfy the $(\mathrm{PS})$ condition and $I(0)=0$. If $\mathcal{B}=V \oplus X$, where $V$ is finite dimensional and I satisfies

(A3) there are constants $\rho, \alpha>0$ such that $\left.I\right|_{\partial B_{\rho} \cap X} \geq \alpha$, and

(A4) for each finite dimensional subspace $\tilde{E} \subset \mathcal{B}$, there is $R=R(\tilde{E})$ such that $I \leq 0$ on $\tilde{E} \backslash B_{R(\tilde{E})}$,

then I has an unbounded sequence of critical values.

\section{Proof of Theorem 1.2}

Now we are going to establish the corresponding variational framework to obtain fast homoclinic solutions of (DS). To this end, define the functional $I: \mathcal{B}=E \rightarrow \mathbb{R}$ by

$$
\begin{aligned}
I(u) & =\int_{\mathbb{R}} e^{Q(t)}\left[\frac{1}{2}|\dot{u}(t)|^{2}+\frac{1}{2}(L(t) u(t), u(t))-W(t, u(t))\right] d t \\
& =\frac{1}{2}\|u\|^{2}-\int_{\mathbb{R}} e^{Q(t)} W(t, u(t)) d t .
\end{aligned}
$$

Lemma 3.1 Under the conditions of Theorem 1.2, we have

$$
I^{\prime}(u) v=\int_{\mathbb{R}} e^{Q(t)}\left[(\dot{u}(t), \dot{v}(t))+(L(t) u(t), v(t))-\left(W_{u}(t, u(t)), v(t)\right)\right] d t
$$

for all $u, v \in E$, which yields that

$$
I^{\prime}(u) u=\|u\|^{2}-\int_{\mathbb{R}} e^{Q(t)}\left(W_{u}(t, u(t)), u(t)\right) d t .
$$

Moreover, I is a continuously Fréchet-differentiable functional defined on E, i.e., $I \in$ $C^{1}(E, \mathbb{R})$.

Proof We firstly show that $I: E \rightarrow \mathbb{R}$. By (1.8), there exist constants $R_{1}>0$ and $M>0$ such that

$$
W(t, u) \leq M|u|^{2} \quad \text { for all }(t, u) \in \mathbb{R} \times \mathbb{R}^{n},|u| \leq R_{1} .
$$


Letting $u \in E$, then $u \in C^{0}\left(\mathbb{R}, \mathbb{R}^{n}\right)$, the space of continuous functions $u$ on $\mathbb{R}$ such that $u(t) \rightarrow 0$ as $|t| \rightarrow+\infty$, i.e., $E \subset C^{0}\left(\mathbb{R}, \mathbb{R}^{n}\right)$. Therefore, there is a constant $R_{2}>0$ such that $|t| \geq R_{2}$ implies that $|u(t)| \leq R_{1}$. Hence, by (3.3), we have

$$
\begin{aligned}
0 & \leq \int_{\mathbb{R}} e^{Q(t)} W(t, u(t)) d t \\
& \leq \int_{-R_{2}}^{R_{2}} e^{Q(t)} W(t, u(t)) d t+M \int_{|t| \geq R_{2}} e^{Q(t)}|u(t)|^{2} d t<+\infty
\end{aligned}
$$

Combining (3.1) and (3.4), we show that $I: E \rightarrow \mathbb{R}$.

Next we prove that $I \in C^{1}(E, \mathbb{R})$. Rewrite $I$ as follows:

$$
I=I_{1}-I_{2}
$$

where

$$
I_{1}:=\frac{1}{2} \int_{\mathbb{R}} e^{Q(t)}\left[|\dot{u}(t)|^{2}+(L(t) u(t), u(t))\right] d t, \quad I_{2}:=\int_{\mathbb{R}} e^{Q(t)} W(t, u(t)) d t .
$$

It is easy to check that $I_{1} \in C^{1}(E, \mathbb{R})$, and we have

$$
I_{1}^{\prime}(u) v=\int_{\mathbb{R}} e^{Q(t)}[(\dot{u}(t), \dot{v}(t))+(L(t) u(t), v(t))] d t .
$$

Thus it is sufficient to show that this is the case for $I_{2}$. In the process we see that

$$
I_{2}^{\prime}(u) v=\int_{\mathbb{R}} e^{Q(t)}\left(W_{u}(t, u(t)), v(t)\right) d t
$$

which is defined for all $u, v \in E$. Let $u \in E$ and suppose the norm of $u$ is $M$, i.e., $\|u\|=M$. Then, by $\left(\mathrm{W}_{2}\right)$, for any $\epsilon>0$, there is $\delta>0$ such that $|x| \leq \delta$ implies that

$$
\left|W_{u}(t, x)\right| \leq \frac{\epsilon}{4 C^{2}(M+1)}|x|,
$$

where $C$ is defined in (2.1). It is well known that

$$
\int_{-R}^{R} e^{Q(t)} W(t, u) d t \in C^{1}\left(W^{1,2}\left([-R, R], \mathbb{R}^{n}\right) ; \mathbb{R}\right)
$$

for any finite $R$. Therefore, there is $\sigma=\sigma(\epsilon, R, u)$ such that $v \in E$ and $\|v\| \leq \sigma$ implies that

$$
\left|\int_{-R}^{R} e^{Q(t)}\left[W(t, u+v)-W(t, u)-\left(W_{u}(t, u), v\right)\right] d t\right| \leq \frac{\epsilon}{4}\|v\|
$$

Choose $R$ so large that $|u(t)| \leq \delta / 2$ for $|t| \geq R$. For $v \in E,\|v\| \leq \min \left\{\sqrt{e_{0} \sqrt{\beta}} \delta / \sqrt{2}, 1\right\}$, by (2.4), we have

$$
\|v\|_{\infty} \leq \frac{1}{\sqrt{2 e_{0} \sqrt{\beta}}}\|v\|
$$


Therefore,

$$
\|v\|_{\infty} \leq \frac{\delta}{2}
$$

The mean value theorem, (3.6), and (3.9) show that, for $|t| \geq R$,

$$
|W(t, u+v)-W(t, u)| \leq \frac{\epsilon(|u|+|v|)}{4 C^{2}(M+1)}|v| .
$$

Hence, by (2.1) and Hölder's inequality, one deduces that

$$
\begin{aligned}
\int_{|t|>R} e^{Q(t)}|W(t, u+v)-W(t, u)| d t & \leq \frac{\epsilon}{4 C^{2}(M+1)}\left(\int_{|t|>R} e^{Q(t)}(|u|+|v|)^{2} d t\right)^{\frac{1}{2}}\|v\|_{2, Q} \\
& \leq \frac{\sqrt{2} \epsilon}{4}\|v\|
\end{aligned}
$$

Likewise, by (3.6) and Hölder's inequality, we obtain

$$
\int_{|t|>R} e^{Q(t)}\left|\left(W_{u}(t, u), v\right)\right| d t \leq \frac{\epsilon}{4 C^{2}(M+1)} \int_{|t|>R} e^{Q(t)}\left|u\left\|v \mid d t \leq \frac{\epsilon}{4}\right\| v \|\right.
$$

which together with (3.8) and (3.10) yields the Fréchet differentiability of $I_{2}$. To prove that $I_{2}^{\prime}$ is continuous, suppose that $u_{j} \rightarrow u$ in $E$ and note that

$$
\begin{aligned}
\sup _{\|v\|=1}\left|I_{2}^{\prime}\left(u_{j}\right) v-I_{2}^{\prime}(u) v\right| & =\sup _{\|v\|=1}\left|\int_{\mathbb{R}} e^{Q(t)}\left(W_{u}\left(t, u_{j}(t)\right)-W_{u}(t, u(t)), v(t)\right) d t\right| \\
& \leq \sup _{\|v\|=1}\left\|W_{u}\left(\cdot, u_{j}(\cdot)\right)-W_{u}(\cdot, u(\cdot))\right\|_{2, Q}\|v\|_{2, Q} \\
& \leq C\left\|W_{u}\left(\cdot, u_{j}(\cdot)\right)-W_{u}(\cdot, u(\cdot))\right\|_{2, Q}
\end{aligned}
$$

Let $\epsilon>0$ and choose $R$ so that $|t| \geq R$ implies that

$$
\left|W_{u}(t, u)\right| \leq \frac{\epsilon}{2 C}|u|
$$

Moreover, we can also assume (3.12) holds for $u_{j}$ for large $j$. Therefore, by (3.12), one has

$$
\begin{aligned}
\left(\int_{\mathbb{R}} e^{Q(t)}\left|W_{u}\left(t, u_{j}\right)-W_{u}(t, u)\right|^{2} d t\right)^{\frac{1}{2}} \leq & \left(\int_{-R}^{R} e^{Q(t)}\left|W_{u}\left(t, u_{j}\right)-W_{u}(t, u)\right|^{2} d t\right)^{\frac{1}{2}} \\
& +\frac{\epsilon}{2}\left(\left\|u_{j}\right\|+\|u\|\right)
\end{aligned}
$$

which with (3.11) implies that $I_{2}^{\prime}$ is continuous. Therefore, we show that $I \in C^{1}(E, \mathbb{R})$.

Lemma 3.2 Suppose that $(\mathrm{L}),\left(\mathrm{W}_{2}\right)$, and $\left(\mathrm{W}_{3}\right)$ are satisfied. If $u_{j} \rightarrow u$ (weakly) in $E$, then there exists one subsequence still denoted by $\left\{u_{j}\right\}_{j \in \mathbb{N}}$ such that $W_{u}\left(t, u_{j}\right) \rightarrow W_{u}(t, u)$ in $L^{2}\left(e^{Q(t)}\right)$. 
Proof Assume that $u_{j} \rightarrow u$ in $E$. Then there exists a constant $M>0$ such that, by the Banach-Steinhaus theorem and (2.4),

$$
\sup _{j \in \mathbb{N}}\left\|u_{j}\right\|_{\infty} \leq M, \quad\|u\|_{\infty} \leq M
$$

which combined with (1.9) deduces that there is a constant $d$ (dependent on $M$ ) such that

$$
\left|W_{u}\left(t, u_{j}\right)\right| \leq d\left|u_{j}\right|, \quad\left|W_{u}(t, u)\right| \leq d|u|
$$

for all $j \in \mathbb{N}$ and $t \in \mathbb{R}$. Hence, we have

$$
\begin{aligned}
\left|W_{u}\left(t, u_{j}(t)\right)-W_{u}(t, u(t))\right| & \leq d\left(\left|u_{j}(t)\right|+|u(t)|\right) \\
& \leq d\left(\left|u_{j}(t)-u(t)\right|+2|u(t)|\right) .
\end{aligned}
$$

On the other hand, by Lemma $2.1, u_{j} \rightarrow u$ in $L^{2}\left(e^{Q(t)}\right)$, which yields that there exists one subsequence, still denoted by $\left\{u_{j}\right\}_{j \in \mathbb{N}}$ such that

$$
\sum_{j=1}^{+\infty}\left\|u_{j}-u\right\|_{2, Q}<+\infty
$$

Therefore, $u_{j}(t) \rightarrow u(t)$ for almost every $t \in \mathbb{R}$ and

$$
\sum_{j=1}^{+\infty}\left|u_{j}(t)-u(t)\right|=v(t) \in L^{2}\left(e^{Q(t)}\right) .
$$

Consequently, we have

$$
e^{Q(t)}\left|W_{u}\left(t, u_{j}(t)\right)-W_{u}(t, u(t))\right|^{2} \leq d^{2} e^{Q(t)}(v(t)+2|u(t)|)^{2} .
$$

Using Lebesgue's convergence theorem, the lemma is proved.

Lemma 3.3 If $(\mathrm{L}),\left(\mathrm{W}_{1}\right),\left(\mathrm{W}_{2}\right)$, and $\left(\mathrm{W}_{3}\right)$ hold, then I satisfies the (PS) condition.

Proof Assume that $\left\{u_{j}\right\}_{j \in \mathbb{N}} \subset E$ is a sequence such that $\left\{I\left(u_{j}\right)\right\}_{j \in \mathbb{N}}$ is bounded and $I^{\prime}\left(u_{j}\right) \rightarrow 0$ as $j \rightarrow+\infty$. Then there exists a constant $M>0$ such that

$$
\left|I\left(u_{j}\right)\right| \leq M, \quad\left\|I^{\prime}\left(u_{j}\right)\right\|_{E^{*}} \leq M
$$

for every $j \in \mathbb{N}$.

We firstly prove that $\left\{u_{j}\right\}_{j \in \mathbb{N}}$ is bounded in $E$. By (3.1), (3.13), and $\left(\mathrm{W}_{1}\right)$, we obtain that

$$
\begin{aligned}
\left(\frac{\mu}{2}-1\right)\left\|u_{j}\right\|^{2}= & \mu I\left(u_{j}\right)-I^{\prime}\left(u_{j}\right) u_{j} \\
& +\int_{\mathbb{R}}\left(\mu W\left(t, u_{j}(t)\right)-\left(W_{u}\left(t, u_{j}(t)\right), u_{j}(t)\right)\right) d t \\
\leq & \mu M+M\left\|u_{j}\right\| .
\end{aligned}
$$


Since $\mu>2$, inequality (3.14) shows that $\left\{u_{j}\right\}_{j \in \mathbb{N}}$ is bounded in $E$. Then the sequence $\left\{u_{j}\right\}_{j \in \mathbb{N}}$ has a subsequence, again denoted by $\left\{u_{j}\right\}_{j \in \mathbb{N}}$, and there exists $u \in E$ such that

$$
u_{j} \rightarrow u, \quad \text { weakly in } E .
$$

Hence,

$$
\left(I^{\prime}\left(u_{j}\right)-I^{\prime}(u)\right)\left(u_{j}-u\right) \rightarrow 0
$$

as $j \rightarrow+\infty$. Moreover, by Lemma 3.2 and Hölder's inequality, passing to subsequence if necessary, we have

$$
\int_{\mathbb{R}} e^{Q(t)}\left(W_{u}\left(t, u_{j}(t)-W_{u}(t, u(t)), u_{j}(t)-u(t)\right) d t \rightarrow 0 .\right.
$$

On the other hand, an easy computation shows that

$$
\begin{aligned}
\left(I^{\prime}\left(u_{j}\right)-I^{\prime}(u), u_{j}-u\right)= & \left\|u_{j}-u\right\|^{2} \\
& -\int_{\mathbb{R}} e^{Q(t)}\left(W_{u}\left(t, u_{j}(t)-W_{u}(t, u(t)), u_{j}(t)-u(t)\right) d t,\right.
\end{aligned}
$$

which deduces that $\left\|u_{j}-u\right\| \rightarrow 0$ as $j \rightarrow+\infty$.

Now we are in a position to give the proof of Theorem 1.2. We divide the proof into several steps.

\section{Proof of Theorem 1.2}

Step 1 It is clear that $I(0)=0$ and $I \in C^{1}(E, \mathbb{R})$ satisfies the (PS) condition by Lemmas 3.1 and 3.3.

Step 2 We now show that there exist constants $\rho>0$ and $\alpha>0$ such that $I$ satisfies condition (A1) of Lemma 2.3. By (1.9), for all $\varepsilon>0$, there exists $\delta>0$ such that $W(t, u) \leq \varepsilon|u|^{2}$ whenever $|u| \leq \delta$. Choosing $\rho=\frac{\delta}{\sqrt{2 e_{0} \sqrt{\beta}}}$ and $\|u\|=\rho$, we have $\|u\|_{\infty} \leq \delta$. Hence $W(t, u(t)) \leq \varepsilon|u(t)|^{2}$ for all $t \in \mathbb{R}$. Integrating on $\mathbb{R}$ and by (2.1), we get

$$
\int_{\mathbb{R}} e^{Q(t)} W(t, u(t)) d t \leq \varepsilon\|u\|_{2, Q}^{2} \leq \varepsilon C^{2}\|u\|^{2} .
$$

In consequence, combining this with (3.1), we obtain that, for $\|u\|=\rho$,

$$
\begin{aligned}
I(u) & =\frac{1}{2}\|u\|^{2}-\int_{\mathbb{R}} e^{Q(t)} W(t, u(t)) d t \\
& \geq \frac{1}{2}\|u\|^{2}-\varepsilon C^{2}\|u\|^{2} \\
& =\left(\frac{1}{2}-\varepsilon C^{2}\right)\|u\|^{2} .
\end{aligned}
$$

Setting $\varepsilon=\frac{1}{4 C^{2}}$, inequality (3.15) implies that

$$
\left.I\right|_{\partial B_{\rho}} \geq \frac{\rho^{2}}{4}=\alpha>0
$$


Step 3 It remains to prove that there exists $e \in E$ such that $\|e\|>\rho$ and $I(e) \leq 0$, where $\rho$ is defined in Step 2. By (3.1), we have, for every $m \in \mathbb{R} \backslash\{0\}$ and $u \in E \backslash\{0\}$,

$$
I(m u)=\frac{m^{2}}{2}\|u\|^{2}-\int_{\mathbb{R}} e^{Q(t)} W(t, m u(t)) d t .
$$

Take some $\psi \in E$ such that $\|\psi\|=1$. Then there exists a subset $\Omega$ of positive measure of $\mathbb{R}$ such that $\psi(t) \neq 0$ for $t \in \Omega$. Take $m>0$ such that $m|\psi(t)| \geq 1$ for $t \in \Omega$. Then, by (1.7), we obtain that

$$
I(m \psi) \leq \frac{m^{2}}{2}-m^{\mu} \int_{\Omega} \eta(t) e^{Q(t)}|\psi(t)|^{\mu} d t
$$

Since $\eta(t)>0$ and $\mu>2$, (3.16) implies that $I(m \psi)<0$ for some $m>0$ with $m|\psi(t)| \geq 1$ for $t \in \Omega$ and $\|m \psi\|>\rho$, where $\rho$ is defined in Step 2. By Lemma 2.3, I possesses a critical value $c \geq \alpha>0$ given by

$$
c=\inf _{g \in \Gamma} \max _{s \in[0,1]} I(g(s)),
$$

where

$$
\Gamma=\{g \in C([0,1], E): g(0)=0, g(1)=e\} .
$$

Hence there is $u \in E$ such that

$$
I(u)=c \quad \text { and } \quad I^{\prime}(u)=0 .
$$

Step 4 Now suppose that $W(t, u)$ is even in $u$, i.e., $\left(\mathrm{W}_{4}\right)$ holds, which implies that $I$ is even. Furthermore, we have already known that $I(0)=0$ and $I \in C^{1}(E, \mathbb{R})$ satisfies the (PS) condition in Step 1.

To apply Lemma 2.4, it suffices to prove that $I$ satisfies conditions (A3) and (A4) of Lemma 2.4. Here we take $V=\{0\}$ and $X=E$. (A3) is identically the same as in Step 2, so it is already proved. Now we prove that (A4) holds. Let $\tilde{E} \subset E$ be a finite dimensional subspace. From Step 3, we know that, for any $\psi \in \tilde{E} \subset E$ such that $\|\psi\|=1$, there is $m_{\psi}>0$ such that

$$
I(m \psi)<0 \quad \text { for }|m| \geq m_{\psi}>0 .
$$

Since $\tilde{E} \subset E$ is a finite dimensional subspace, we can choose $R=R(\tilde{E})>0$ such that

$$
I(u)<0, \quad \forall u \in \tilde{E} \backslash B_{R} .
$$

Hence, by Lemma 2.4, I possesses an unbounded sequence of critical values $\left\{c_{j}\right\}_{j \in \mathbb{N}}$. Let $u_{j}$ be the critical point of $I$ corresponding to $c_{j}$, then (DS) has infinitely many distinct fast homoclinic solutions.

\section{Acknowledgements}

The authors would like to thank the referees for carefully reading the manuscript, giving valuable comments and suggestions to improve the results as well as the exposition of the paper. 
Funding

The authors are supported financially by the National Natural Science Foundation of China (11771044).

\section{Availability of data and materials}

Data sharing not applicable to this article as no datasets were generated or analysed during the current study.

\section{Competing interests}

The authors declare that there is no conflict of interests regarding the publication of this paper.

Authors' contributions

All authors contributed equally and significantly in writing this article. All authors read and approved the final manuscript.

\section{Publisher's Note}

Springer Nature remains neutral with regard to jurisdictional claims in published maps and institutional affiliations.

Received: 19 March 2018 Accepted: 29 November 2018 Published online: 05 December 2018

\section{References}

1. Alves, C.O., Carrião, P.C., Miyagaki, O.H.: Existence of homoclinic orbits for asymptotically periodic systems involving Duffing-like equation. Appl. Math. Lett. 16(5), 639-642 (2003)

2. Ambrosetti, A., Rabinowitz, P.H.: Dual variational methods in critical point theory and applications. J. Funct. Anal. 14(4), 349-381 (1973)

3. Caldiroli, P., Montecchiari, P.: Homoclinic orbits for second order Hamiltonian systems with potential changing sign. Commun. Appl. Nonlinear Anal. 1(2), 97-129 (1994)

4. Chen, P., Tang, X.H.: Fast homoclinic solutions for a class of damped vibration problems with subquadratic potentials. Math. Nachr. 286(1), 4-16 (2013)

5. Chen, P., Tang, X.H., Agarwal, R.P.: Fast homoclinic solutions for a class of damped vibration problems. Appl. Math. Comput. 219(11), 6053-6065 (2013)

6. Coti Zelati, V., Rabinowitz, P.H.: Homoclinic orbits for second order Hamiltonian systems possessing superquadratic potentials. J. Am. Math. Soc. 4(4), 693-727 (1991)

7. Daouas, A.: Homoclinic solutions for superquadratic Hamiltonian systems without periodicity assumption. Nonlinear Anal. 74(11), 3407-3418 (2011)

8. Ding, Y.H.: Existence and multiplicity results for homoclinic solutions to a class of Hamiltonian systems. Nonlinear Anal. 25(11), 1095-1113 (1995)

9. Ding, Y.H., Lee, C.: Homoclinics for asymptotically quadratic and superquadratic Hamiltonian systems. Nonlinear Anal. 71, 1395-1413 (2009)

10. Izydorek, M., Janczewska, J.: Homoclinic solutions for a class of the second order Hamiltonian systems. J. Differ. Equ. 219(2), 375-389 (2005)

11. Korman, P., Lazer, A.C.: Homoclinic orbits for a class of symmetric Hamiltonian systems. Electron. J. Differ. Equ. 1994, 01 (1994)

12. LV, X., Jiang, J.F.: Existence of homoclinic solutions for a class of second-order Hamiltonian systems with general potentials. Nonlinear Anal., Real World Appl. 13, 1152-1158 (2012)

13. Lv, X., Lu, S.P., Yan, P.: Existence of homoclinic solutions for a class of second-order Hamiltonian systems. Nonlinear Anal. 72, 390-398 (2010)

14. LV, Y., Tang, C.L.: Existence of even homoclinic orbits for a class of Hamiltonian systems. Nonlinear Anal. 67(7), 2189-2198 (2007)

15. Omana, W., Willem, M.: Homoclinic orbits for a class of Hamiltonian systems. Differ. Integral Equ. 5(5), 1115-1120 (1992)

16. Poincaré, H.: Les méthodes nouvelles de la mécanique céleste. Gauthier-Villars, Paris (1897-1899)

17. Rabinowitz, P.H.: Minimax methods in critical point theory with applications to differential equations. In: CBMS Reg. Conf. Ser. in. Math., vol. 65. American Mathematical Society, Provodence, RI (1986)

18. Rabinowitz, P.H.: Homoclinic orbits for a class of Hamiltonian systems. Proc. R. Soc. Edinb., Sect. A 114(1-2), 33-38 (1990)

19. Rabinowitz, P.H., Tanaka, K.: Some results on connecting orbits for a class of Hamiltonian systems. Math. Z. 206(3), 473-499 (1991)

20. Sun, J.T., Chen, H.B., Nieto, J.J.: Homoclinic solutions for a class of subquadratic second-order Hamiltonian systems. J. Math. Anal. Appl. 373(1), 20-29 (2011)

21. Tang, X.H., Lin, X.Y.: Infinitely many homoclinic orbits for Hamiltonian systems with indefinite subquadratic potentials. Nonlinear Anal. 74, 6314-6325 (2011)

22. Tang, X.H., Lin, X.Y.: Existence of infinitely many homoclinic orbits in Hamiltonian systems. Proc. R. Soc. Edinb., Sect. A $141,1103-1119(2011)$

23. Wan, L.L., Tang, C.L.: Existence and multiplicity of homoclinic orbits for second order Hamiltonian systems without (AR) condition. Discrete Contin. Dyn. Syst., Ser. B 15, 255-271 (2011)

24. Wang, J., Zhang, F.B., Xu, J.X.: Existence and multiplicity of homoclinic orbits for the second order Hamiltonian systems. J. Math. Anal. Appl. 366, 569-581 (2010)

25. Wu, X., Chen, S.X., Teng, K.M.: On variational methods for a class of damped vibration problems. Nonlinear Anal. 68, $1432-1441(2008)$

26. Yang, M.H., Han, Z.Q.: The existence of homoclinic solutions for second-order Hamiltonian systems with periodic potentials. Nonlinear Anal., Real World Appl. 12, 2742-2751 (2011)

27. Zhang, C.F., Han, Z.Q.: Infinitely many homoclinic orbits for a class of second-order damped differential equations. Math. Methods Appl. Sci. 38, 5048-5062 (2015) 
28. Zhang, Q.Y., Liu, C.G.: Infinitely many homoclinic solutions for second order Hamiltonian systems. Nonlinear Anal. 72, 894-903 (2010)

29. Zhang, Z.H., Yuan, R.: Homoclinic solutions for a class of non-autonomous subquadratic second order Hamiltonian systems. Nonlinear Anal. 71, 4125-4130 (2009)

30. Zhang, Z.H., Yuan, R.: Homoclinic solutions for a class of asymptotically quadratic Hamiltonian systems. Nonlinear Anal., Real World Appl. 11, 4185-4193 (2010)

31. Zhang, Z.H., Yuan, R.: Fast homoclinic solutions for some second order non-autonomous systems. J. Math. Anal. Appl. 376, 51-63 (2011)

32. Zou, W.M., Li, S.J.: Infinitely many homoclinic orbits for the second-order Hamiltonian systems. Appl. Math. Lett. 16, $1283-1287(2003)$

Submit your manuscript to a SpringerOpen ${ }^{\circ}$ journal and benefit from:

- Convenient online submission

- Rigorous peer review

- Open access: articles freely available online

- High visibility within the field

- Retaining the copyright to your article

Submit your next manuscript at $\gg$ springeropen.com 